Desterr. Botan Zeitsrhint1878.

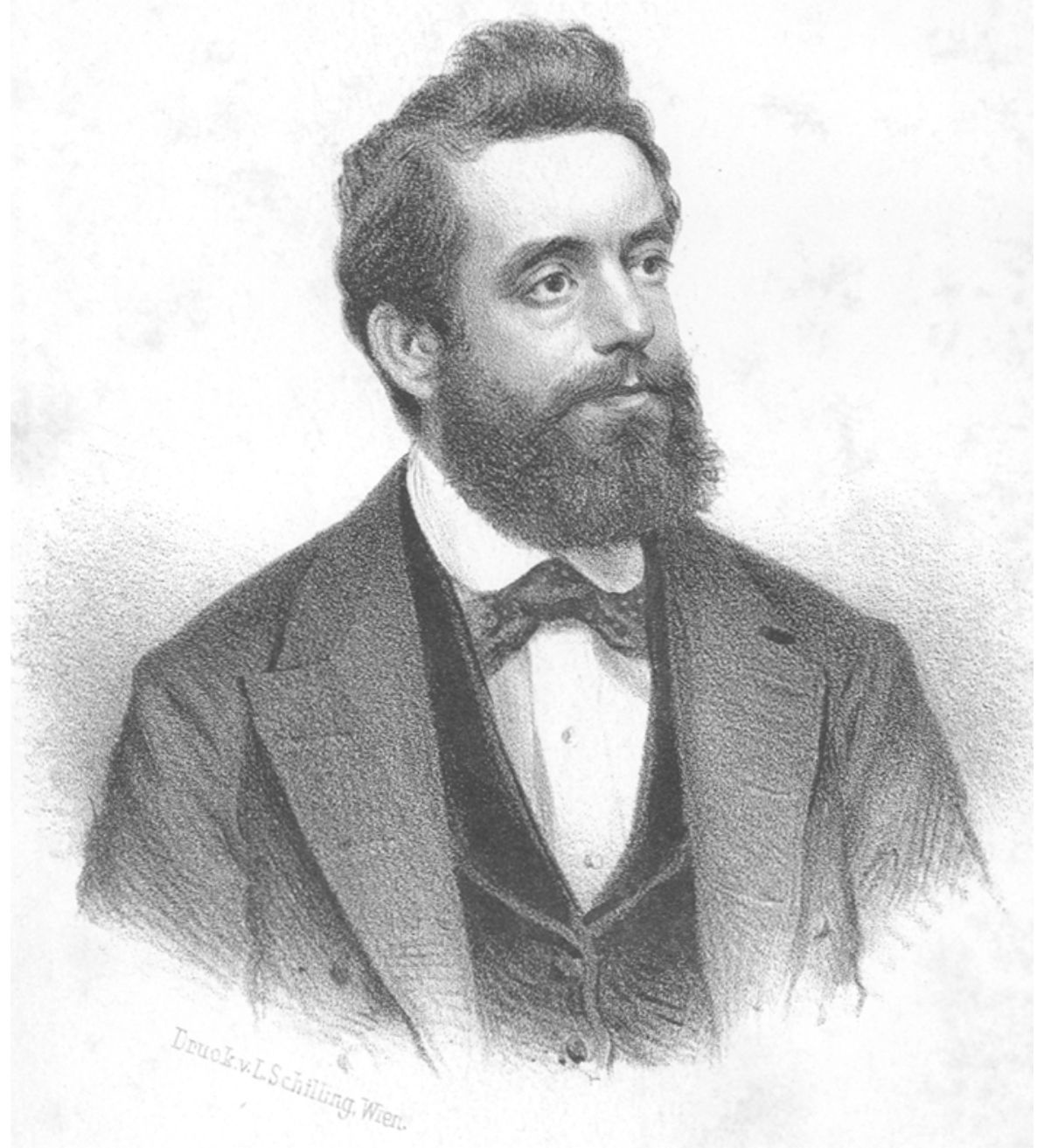

Prof, D. D. Nage. 


\section{Oesterreichische}

\section{Botanische Zeitschrift.}

\section{Gemeinnütziges Organ}

fïr

\begin{abstract}
Die Usterrelchische botanische zeitsehrift erscheint
\end{abstract}

den Ersten jeden Monats. Man pranumerirt auf" selbe

mit 8. 1. ost. W (16 R. Mark.) ganz jährig, oder mit 4 f. $6 . W \cdot(8 R . M a r k)$ ha 1 bjährig. Inserate

die ganze Petitzeile $15 \mathrm{kx}$. öst. W.

\section{Botanik und Botaniker,}

Gärtner, Oekonomen, Forstmänner, Aerte, Apohteker und Techniker.

$\mathrm{N}_{=}^{\circ} \mathbf{1}$.
Exemplare

die frel dutch die Post bezogen werden sollen, sind bios bei der Redaktloa (V. Bez., Schlossgasse Nr. 15) su pränumeriren. Im Wege des Buchhandels übernimmt Pränmeration. C. Gerold's Sohn in Wien,

sowie alle übrigen Buchhandlungen.

\section{Jahrgang. Jänner $18 \% 8$.}

INARALT: A Vogl. Von Dr. Wiesner. - Zapfengallen an Fichtenzweigen. Von Winkler. -

Vegetations-Verhältaisse. Von Dr. Kerner. - Vorkommen koagulirten Milchsaftes. Von Höhnel. - Drei Cerastien. Von Stein. - Silene Ungeri. Von Dr. Heldreich. - Pdanzen auf der Welt-

aussteliung. Voll Ant o in e. - Berichtigung. - Literaturberichte, - Correspondenz. Von Dr. B or bás.

Evers. - Personalnotizen. - Botanscher Tauschverein. - Inserate.

\section{Gallerie österreichischer Botaniker. XXII. August Emil Vogl. \\ (Mit einem lithographirten Portiät.)}

Die Kunst des Biographen zeigt sich so oft in dem ersten Satze seiner Einleitung: ein geistreicher Gedanke in Beziehung zu dem Leben, welches geschildert werden soll, gebracht, erweckt rasch das Interesse für den Mann, um den es sich handelt; und gerne folgt der Leser dem Erzähler auch dann, wenn die Schicksale einer nur wenig gekannten Person entrollt werden sollten.

Wenn ich auch diese Kunst, die Arago in so wundervoller Weise übte, besässe, so brauchte ich sie doch nicht auszuüben, indem ich mich anschicke, August Vogl's Lebenslauf niederzuschreiben. Sein Name, über die Grenzen des Vaterlandes hinaus weit gekannt und geachtet, ist jedem österr. Botaniker geläufig, und jeder mit der Anatomie der Pflanzen und der angewandten Botanik Vertraute, weiss, dass Vogl als Anatom sich einen geachteten Ruf erwarb, dass er zu den bedeutendsten europäischen Pharmakognosten zählt und in der Anwendung der Pflanzenanatomie auf Pharmakognosie wohl als die erste jetzt lebende Auloritat genannt zu werden verdient. 
So knüpft sich also des Interesses genug an diesen Namen, auf dass man seines Trägers Entwicklungsgang gerne und ohne besondere Anregung folge.

August Emil Vogl wurde am 3. August 1833 zu Weisskirchen in Mähren geboren. Sein Vater, Apotheker dortselbst, ertheilte allen seinen Kindern eine liebevolle Erziehung, und suchte in allen Sinn für die Natur, für ihre Schönheiten sowohl wie für ihre Produkte und ihre Erscheinungen zu wecken. Wenn dieso Anregungen auch bei allen Kindern des wackeren und geachteten Weisskirchener Bürgers Eindruck gemacht haben mochten; eine Bedeulung für's Leben hatten sie nur für den kleinen August, der schon in früher Kindheit Pflanzen, Mineralien u. s. w. sammelte, als Gymnasiast schon der vielleicht kenntnissreichste Florist von Olmütz war, und als er bald darauf die medizinischen Studien in Wien begann, schon bei dor Einfübrung in die botanischen Kreise der Residenz kein Unbekannter mehr war.

Doch ich muss zu dem kleinen August nochmals zurück. Sein Vater war von der Wichtigkeit der realen Studien so überzeugt, dass er, bevor er seinen Sohn auf das Gymnasium schickte, ihn zuersi die damalige sogenannte vierte Klasse (welche etwa den unteren Klassen der heutigen Unterrealschule entsprach) durchmachen liess. 1846 trat V. in das Olmülzer Gymnasium ein, und zeichnete sich dort fast in allen Lehrfächern aus. Mit gleichstrebenden Kollegen (z. B. mit A. Makowsky, nunmehr Professor an der technischen Hochschule zu Brünn) botanisirte or in der näheren und weiteren Ungebung von Olmütz, Weisskirchen und Kremsier, und trat mit anderen jungen Botanikern (u. a. mit dem Schreiber dieser Zeilen, der damals in Brünn studirte) in botanischen Tausch- und Briefverkehr. Schon in dieser Zeit machte er seine ersten Versuche als botanischer Schriftsteller und veröffentlichte u. A. eine Flora von Olmütz in diesen Blättern.

Im Jahre 1854 legte $V$. die Maturitätsprüfung am Olmützer Gymnasium mit Auszeichnung ab und bezog den höheren Lehrliurs der 1854 wieder eröffneten und neu eingerichteten medizinischchirurgischen Josefs-Akademie in Wien. Als er mit den Vorbereitungen zu den medizinischen Rigorosen beschäftigt war, brach der italienisch-österreichische Krieg aus. V. wurde, noch bevor er zum Doktor der Medizin promovirt wurde, als provisorischer Oberarzt auf den Kriegsschauplatz gesendet, wo er auf dem Verhandplatze zu Nabresina und in den Lazarethen zu Mantua eine Aufgabe zu lösen hatte, wie eine solche wohl nur selten einem so jungen Feldarzt zufallen wird. Die traurigen Erinnerungen an diese feldärztlichen Lorbern, die ihm zu pflücken um so herber ankam, als er weniger Neigung zum ärztlichen Stande als für eine reine wissenschaftliche. Thätigkeit empfand; ich sage die traurigen Erinnerungen an jene kriegschirurgische Thätigkeit werden reichlich anfgewogen durch die Nachempfindung seiner ersten und letzten Liebe. In Mantua lernte $V$. jenes schöne, edle Mädchen italienischer Abkunft kenneri, welches er ein 
Jahr später als seine Frau heimführte und mit der er in glücklichster nunmehr von vier Kindern gesegneter Ehe lebt.

Nach Beendigung des Krieges nach Wien zurückgekehrt erwarb er an der Josefs-Akademie den Grad eines Doktors der gesammten Heilkunde und damit den Rang eines Oberarztes. Ein glückliches Geschick brachte ihn in eine seinen Neigungen entsprechende Richtung. Er wurde am Josefinum Assistent bei der Lehrkanzel der Naturgeschichte (unter Prof. C. v. Ettingshausen) und konnte nun sein Lieblingsfach - Botanik - nach Herzenslust treiben. Zwischen V. und dem Schreiber dieser Zeilen entwickelte sich schon damals ein reger Verkehr, der auf der Basis wahrer Freundschaft sich aufbaute, aber doch hauptsächlich um die Anatomie der Pflanzen sich drehte. Fünf Jahre blieb Vogl in dieser bescheidenen Stellung. 1864 habilitirte er sich als Privatdozent für Pharmakognosie an der medizinischen Fakultät der Wiener Universität.

Im Jahre 1866 erfolgle Vogl's Beförderung zum Regimentsarzt. In diesem und dem folgenden Jahre war er allerdings in verschiedenen Militärspitalern praklisch beschäftigt, ohne aber seine theoretischen Arbeiten beiseite gelassen zu haben. Im Gegentheile; 1867 erschien sein Werk über die Chinarinden des Wiener Handels und der Wiener Sammlungen, eine gründliche mühevolle Arbeit, welche zeigt, mit welcher Hingebung er seine wissenschaftlichen Ziele verfolgte, selbst in einer Zeit, wo er seine beste Kraft der Ausfüllung seines ärztlichen Berufes widmen musste. Diese werthvolle monographische Arbeit ist auch desshalb interessant, weil aus ihr hervorgeht, wie tief der Autor in der anatomischen Behandlung der Droguen bereits vordrang, was der hervorragendste zeitgenössische, kürzlich verstorbene Chinologe Weddel, mit Rücksicht auf diese Arbeit und in anerkennendster Weise hervorhob.

In dem genannten Jahre erhielt V. die Stelle eines Bibliothekars am Josefinum. Er benützte diese Gelegenheit, un seine Literaturkenntnisse zu erweitern und scheute nicht die Mühe, die reiche Büchersammlung vom Grunde auf neu aufzustellen und zu inventarisiren. Zwei Jahre später erfolgte seine Ernennung zum Adjunkien am chem. Laboratorium des damaligen Prof., jelzigen Hofrath und Sanitälsreferenten im Ministerium des Innern, Dr. Schneider, wo er mit der Durchführung gerichtlich-chemischer und mikroskopischer Untersuchungen betraut wurde. In diese Zeit fälit die mit Schneider gemeinschaftlich durchgeführte Herausgabe des hlassischen Kommentars zur österr. Pharmakopöe, für welche Vogl den naturgeschichtlichen, Schneider den chemischen Theil bearbeitete.

Im Frühjahre 1870 folgte $V$. einem Rufe als Professor der Botanik und Zoologie an das deutsche Polytechnikum in Prag, woselbst er ausser den obligaten Vorlesungen noch regelmässig Vorträge über technische Waarenkunde und Mikroskopie hielt, die sich eines solchen Erfolges zu erfreuen halten, dass ihm über Vorschlag des Professorenkollegiums vom böhm. Landesausschusse eine namhafte Personalzulage bewilligt und nicht lange darauf von $\mathrm{Sr}$. Majestät der 
Titel eines ordentlichen Professors "in wohlverdienter Anerkennung vorzüglicher Leistungen" verliehen wurde.

Im Jahre 1872 hatte $\mathrm{V}$. die Redaktion der naturwiss. Zeitschrift "Lotos" ibernommen und durch vier Jahr geführt. Wie er diess that, geht am besten aus dem Dankschreiben hervor, welches das Präsidium des Vereines "Lolos" unter dem 27. Dezember 1875 an ihn richtete. Darin heisst es: „In der letzten diessjährigen Versammlung des Vereines "Lotos" vom 15. l. M. wurde ich beauftragt, Ihnen im Namen des Vereines den aufrichtigsten Dank auszusprechen für die durch so lange Zeit mit unermüdeter Hand und in trefflichster Weise besorgte Herausgabe der Zeitschrift. Es wurde in der Versammlung hervorgehoben, dass vier volle Jahre verstrichen sind, seit Sie die Güte hatten, die Redaktion zu übernehmen, dass Sie es vermochten, den Blättern tüchtige Originalartikel zuzuführen, darunter nicht wenige aus lhrer Feder stammend, und den Inhalt und Umfang der Schriften zu erweitern. Es wurde allgemein und dankbarst anerkannt, dass unter Ihrer Leitung die Zeilschrift "Lotos" ihre glänzendste Periode fand, und dass es nur Ihrer Kraft und Ausdauer gelingen konnte, unter schwierigen Verhältnissen des Redaktionsamtes in so ausgezeichneter Weise zu walten."

Die unermüdliche wissenschaftliche und lehramtliche Thätigkeit V's. verbreitete seinen Ruf im weiten Kreise und so ereignete sich der seltene Fall, dass er im Frühlinge des Jahres 1874 zwischen zwei ehrenvollen Berufungen zu wählen hatte. Das Professorenkollegium der forstlichen Hochschule zu Mariabrumn berief ihn auf die, durch die Ernennung des Schreibers dieser Zeilen zum Professor an der Universilät erledígte Lehrkanzel der Botanik, und das Professorenkollegium der Wiener medizinischen Fakultät an Stelle des Professors und Hofrathes Dr. v. Schroff zum ordentl. Professor der Pharmakognosie und Pharmakologie. Die Entscheidung war leicht zu treffen. Durch Uebernahme des letztgenannten Lehramtes, war der als Pflanzenanatom wie als Pharmakognost gleich ausgezeichnete Mann am Ziele seiner Wünsche. Dennoch schied er schweren Herzens von seinen theuren ihn verehrenden Prager Kollegen. Bei seinem Abgange von Prag drückte ihm der Landesausschuss des Königreichs Böhmen "für die ausgezeichnete und erfolgreiche Thätigkeit am deutschen polytechnischen Landesinstitute die wohlverdiente Anerkennung" aus.

In Wien war $V$, bald wieder heimisch geworden und erwarb sich rasch das Vertrauen seiner neuen Kollegen, was sich wohl darin schon ausspricht, dass er bereits im zweiten Jahre seiner hierortigen lehramtlichen Thätigkeit zum Prodekan, im nächstfolgenden zum Dekan gewählt und im laufenden Studienjahr zum Examinator bei den Prüfungen der Aerzte zur Erlangung einer bleibenden Anslellung im öffentlichen Sanitätsdienste ernannt wurde.

Von der wissenschaftlichen Thätigkeit V's. gibt am besten das nachfolgende Verzeichniss seiner botanischen und pharmakognostischen 
Arbeiten ein Bild. Alle seine Untersuchungen zeichnen sich durch Gründlichkeit und völlige Beherrschung des Stoffes, die Darstellung durch Einfachheit und Klarheit aus.

\section{Publikationen.}

A Selbständig erschienen:

1. Die Chinarinden des Wiener Handels und der Wiener Sammlungen. Mai 1867.

2. Mit F C. Schneider: Kommentar zur österr. Pharmakopöe 2. Aufl. 1. Band. Arzneikörper aus den drei Naturreichen. Wien 1869. (Im Laufe des Jahres 1878 erscheint eine 3. Auflage des Kommentars).

3. Nahrungs- und Genussmittel aus dem Pflanzenreiche. Wien 1872 (In französischer Uebersetzung von A. Focillon. Paris 1876 erschienen).

B. Aufsätze und Abhandlungen in Zeitschriften, Gesellschaftschriften etc.

A. In der österr. bot. Zeitschrift.

Mehrere Aufsätze in den Jahren 1853-1856.

B. In den Schriften der k. k. zoolog. bot. Ges.

1863. Beiträge zur Anatomie und Histologie der unterirdischen Theile von Convovulus arvensis.

1864. Zur näheren vergleichend histologischen Kenntniss des Bitterholzes (Lignum Quassiae).

1865. Beiträge zur Kenntniss der Entstehung krystallinischer Bildungen im Inhalte der Pflanzenzelle.

1869. Beiträge zur Pflanzenanatomie: I. die Milchsaftorgane der Cinchonen; II. die Siebröhren der Cinchonen; III. ein Beilrag zur Kenntniss der Krystalloide.

1876. Beiträge zur Kenntniss der sogenannten falschen Chinarinden (in der Festschrift der Gesellsch.)

C. In Pringsheim's Jahrb. für wiss. Botanik:

1866. Beiträge zur Kenntniss der Milchsaftorgane der Pflanzen.

1874. Ueber den Bau des Holzes von Ferreira spectabilis und die Bildungsweise des sogen. Angelin-Pedraharzes.

D. In der botan. Zeitung:

1866. Ueber den körnigen Zelleninhalt im Wurzelstocke und im Stempel von Spiraea Ulmaria L. und über Harzkörner in der Rinde von Portlandia grandiflora.

1866. Ueber Milchsaftgefässe in der Klette.

E. In den Silzungsberichten der $k$. Akad. der Wiss.

1863. Ueber die Intercellularsubstanz und die Milchsaftgefässe in der Wurzel des gemeinen Löwenzahns.

1864. Phytohistologische Beiträge. I. Kamala, IJ. die Blätter der Sarracenia purpurea.

1866. Ueber das Vorkommen von Gerb- und verwandten Stoffen in unterirdischen Pflanzentheilen.

F. In der Wiener landwirthschaftlichen Zeitung:

1868. Zur Naturgeschichte der Krappwurzel. 
G. In der Zeitschrift "Lotos":

1871. Zur Kenntniss der chinesischen Gelbbeeren.

1872. Kurze Mittheilung über einige histologische und histochemische Verhältnisse des Wau's (Reseda Luteola).

1873. Untersuchungen über den Bau und das mikrochemische Verhalten der wichtigsten Farbhölzer des Handels.

1875. Ueber Tamarisken-Gallen.

H. In den mediz. Jahrbüchern.

1864. Zur näheren Kenntniss der Turbithwurzel des Handels und ilhrer Harze, (Habililationsschrift).

1865. Ueber blutstillend wirkende Spreuhacare der Farne.

1866. Pharmakognostische Studien uber die Granalbaumrinde (Gekrönte Preisschrift).

I. In der Zeitschrift des Allg. öst. Apoth.-Vereines.

Histologisch pharmakognostische Notizen.

1864. I. Scammonium II. Traganth III. Semen tonco.

1865. IV. Zur Kenntniss der Entstehung des Ammoniakharzes. 1866-1867 V. Folia Coca. VI. Chinologische Beiträge.

1865. Histologische Studien über den Bau und die chemische Zusammensetzung der Seifenwurzeln.

1867. Zur Pharmakognosie der Ipecacuanha.

1868. Pharmakognostische Beiträge. 1. Busch-Thee. 2. Cupido-

Rinde. 3. Scuson-Rinde. 4. Musena-Rinde. 5. Alkornok-Rinde.

6. Eine falsche Chinarinde.

1870. Sasaparilla-Diagnosen.

1871. Zur Pharmakognosie einiger weniger bekannten Rinden. (29. Rinden anat. besehen).

1872. Condurango-Rinde.

1872. Pharmakognostische Beitrage. lpecacuanha de Costa ricca. 1873. Cortex Remigiae.

K. im neuen Jahrbuch für Pharmazie.

1870. Untersuchung der Chinarinden auf ihren Gehalt an Alkaloïden.

Man sieht aus diesem reichhaltigen Verzeichnisse, dass 'des Autors Hauptziel eine möglichst weitgehende anatomische Vertiefung der Pharmakognosie ist. Ein tieferer Einblick in seine Arbeiten lehrt ferner, wie richtig er seine Aufgabe erfasste, indem er sich nie von der reinen Pllanzenanatomie gotrennt hat, wohl erkennend, dass nur die stete Verbindung mit der reinen Wissenschaft es möglich macht, in der angewandten wahrhaft grosse Resultate zu erzielen.

Nach einer so eminenten wissenschaftlichen Bethätigung konnle vielfache aussere Anerkennung seiner Verdienste nicht ausbleiben. Zahlreiche wissenschaftliche Vereine und Gesellschaften ernannten ihn zum Mitgliede, beziehungsweise Ehrenmitgliede. Der Jahrgang 1870 der Zeilschrift des Allg. Apotheker-Vereines wurde ihm und Hofr. Schneider gewidmet. Er wurde in die internationale Commission, welche mit der Redaktion einer Pharmacopoea europaea betraut ist, gewählt. 
Vogl's Charakter ist schlicht, offen, vom Grunde aus herzlich, von strengster Ehrenhaftigkeit und peinlichster Gewissenhaftigkeit. Jeder, der ihn, den bedeutenden und doch so bescheidenen Mann kennt, begreift es rasch, wie gross die Zahl seiner Freunde und Verehrer ist, und dass gerade ihm das seltene Los zu Theil wurde, keinen Feind, keinen Gegner, ja vielleicht nicht einmal einen Neider zu haben.

J. Wiesner.

\title{
Kleinere Arbeiten des pflanzenphysiologischen Institutes der Wiener Universität.
}

\author{
XIII.

\begin{abstract}
Lur Anatomie der durch die Fichtenrindenlaus an Fichtenzweigen entstehenden Lapfengallen.
\end{abstract}

Yon Wilibald Winkler.

Die ananasartigen Gallen an den jungen Zweigen der Fichte verdanken ihr Entstehen den Rüsselstichen von Chermes viridis Ratzebg. und Chermes coccineus Ratzebg. Nach Leuckart bohrt sich ein überwinterndes Weibchen an der Stammknospe fest und legt im Frühjahr zahlreiche Eier an dieselbe ab. Die ausgeschlüpften Jungen wiederholen mit ihren Rüsseln die Thätigkeit der Mutter an den -sich entwickelnden Nadeln, die nun in Folge des fortwährenden Reizes in ihren untern Partien bedeutend anschwellen und durch enges Aneinanderschliessen die Gallen bilden, im obern Theil aber normal bleiben. Zwischen je 3 Nadeln findet sich in der Galle eine Höhlung mit schmalem, halbmondförmigen Eingange, in ihr halten sich die Insekten auf. - Wie zu erwarten ist, bieten die durch die Gallenbildung hervorgerufenen Veränderungen in den Geweben (des Zweiges und) der Nadeln einige bemerkenswerthe Eigenthümlichkeiten, die hier in Kurzem erwähnt seien.

An der Oberhaut vermisst man vor Allem die Spaltöffnungen, die nur zuweilen an der Oberseite in sebr reduzirter Zahl und Entwicklung vorkommen, sich aber an dem Nadeltheil über der Galle wieder regelmässig einstellen. Dagegen treten zahlreiche haarartige, oft 2- und mehrzellige Papillen auf, die an den Rändern der Bruthöhlen zu langen, sehr dicht stehenden, mit einem rothen die Reaktionen des Anthocyan zeigenden Farbsloffe erfüllten Haaren werden. Sie greifen hier von beiden Seiten über der Oeffnung in einander und nehmen nach dem Innern der Höhle zu rasch an Grösse ab. Einfach konvexe Zellen, ohne Papillen, setzen die Oberhaut der Höhlen selbst zusammen. - Ferner fällt an der Oberhaut das Auftreten von Stärke in die Augen. Man findet sie in zerstreuten 